\title{
Strahlenschutzausbildung für Ärzte mit Röntgentätigkeit
}

\section{J. Roth ${ }^{a}$, J.-F. Valley ${ }^{b}$ \\ a Radiologische Physik, Universitätsspital Basel \\ b Institut de radiophysique} appliquée, Université de Lausanne

1 Strahlenschutzgesetz (1991) und Strahlenschutzverordnung (1994)

2 Strahlenschutz-Ausbildungsverordnung (1998)

3 Giger M. Röntgen: Was muss ich was darf ich? Schweiz Ärztezeitung 2002;83(44);2333-4.

4 BAG. Ausbildungen im Strahlenschutz und die erlaubten Tätigkeiten im Bereich der Humanmedizin. www.bag.admin.ch/ strahlen/formation/general/d/

5 Aufgaben und Pflichten des Sachverständigen im Bereich der Anwendung ionisierender Strahlung. Merkblatt R-03-04 des BAG (2004).

Korrespondenz:

Prof. Dr. phil. nat. Jakob Roth

Radiologische Physik

Universitätsspital Base

CH-4031 Basel

Tel. 0612653141

Fax 0612653135

jroth@uhbs.ch
Die schweizerische Strahlenschutzgesetzgebung $[1,2]$ schreibt vor, dass Ärzte (gemeint sind im folgenden stets auch die Ärztinnen) eine entsprechende Ausbildung mit Prüfung absolvieren müssen, wenn sie Röntgenuntersuchungen am Menschen durchführen wollen [3, 4]. Die Anforderungen sind zweischichtig. Einerseits betreffen sie die praktische Fertigkeit bei der Anwendung von Röntgengeräten, die sogenannte Sachkunde (das Handwerk). Andererseits regeln sie in einem Betrieb die Anforderungen an den Strahlenschutzsachverständigen (die Verantwortung).

\section{Sachkunde für dosisintensive Röntgenuntersuchungen}

Mit dem eidgenössischen Arztdiplom wird auch die Sachkunde für Röntgenuntersuchungen im Niedrigdosisbereich erworben. Das heisst, dass Röntgenaufnahmen des Thorax, der Extremitäten und des Schädels sowie Durchleuchtungen der peripheren Extremitäten inklusive Ellbogen bzw. oberes Sprunggelenk von jedem Arzt durchgeführt werden dürfen. Der Gesetzgeber geht offensichtlich davon aus, dass dies während des Medizinstudiums erlernt und geübt wird.

Für dosisintensive und interventionelle diagnostische Anwendungen muss zusätzlich die entsprechende Facharztausbildung FMH nachgewiesen werden. Als dosisintensiv gelten Röntgenaufnahmen des Achsenskeletts, des Beckens und des Abdomens, Röntgenuntersuchungen mit mehreren Schnitten (CT, Tomographien) sowie Durchleuchtungen, durchleuchtungsgestützte Kontrastmitteluntersuchungen und Interventionen. In den Weiterbildungsordnungen der betroffenen Fachgesellschaften ist festgelegt, dass dafür eine zweiteilige Ausbildung für die Röntgentätigkeit absolviert werden muss. Abhängig vom Facharztgebiet muss eine Anzahl von bestimmten Röntgenuntersuchungen unter Leitung eines erfahrenen Mentors (Facharzt) durchgeführt und testiert werden. Zusätzlich muss ein praktisch-theoretischer Strahlenschutzkurs absolviert und eine Prüfung bestanden werden.

Diese Regelung gilt für Facharztanwärter nach den neuen Weiterbildungsordnungen. Frühere
Absolventen müssen die Sachkunde für dosisintensive Röntgenuntersuchungen nicht nachholen (Besitzstand). Für die Radiologie, Nuklearmedizin, Radio-Onkologie und Dermatologie gelten andere Anforderungen an die Sachkundeausbildung.

\section{Sachverstand im Strahlenschutz}

Die Strahlenschutzverordnung [1] schreibt vor, dass in jedem Betrieb mit Röntgentätigkeit mindestens ein Arzt die Funktion des Strahlenschutzsachverständigen wahrnimmt und die dafür notwendige Ausbildung [2] absolviert hat. Diese besteht aus einem fünftägigen Kurs mit Prüfung. In einer Einzelpraxis mit Röntgentätigkeit muss sich also der Arzt zum Sachverständigen ausbilden, auch wenn z.B. nur Thorax- und Extremitätenaufnahmen erstellt werden (Niedrigdosisbereich). In einer Mehrfachpraxis und in einer Spitalabteilung mit Röntgenbetrieb muss mindestens einer der Ärzte als Sachverständiger im Strahlenschutz bezeichnet und ausgebildet werden. Er ist verantwortlich, dass die Strahlenschutzvorschriften im Betrieb eingehalten werden und dass die Mitarbeiter entsprechend ausgebildet sind. Das BAG hat kürzlich ein nützliches Merkblatt mit den Pflichten eines Sachverständigen erstellt [5].

Im Gegensatz zur Sachkunde gab es beim Sachverstand keinen Besitzstand. Inhaber mit einer Bewilligung für den Röntgenbetrieb vor Oktober 1994 mussten bis September 2004 den Sachverstand entweder in einer Prüfung des BAG nachweisen oder den fünftägigen Kurs mit Prüfung absolvieren. Eine Betriebsbewilligung erteilt das BAG seit 1995 nur, wenn die entsprechende Person als Sachverständige bezeichnet und in einem Strahlenschutzkurs ausgebildet ist [4]. Für die Radiologie, Nuklearmedizin, RadioOnkologie und Dermatologie gelten andere Anforderungen an den Sachverstand.

\section{Sachkunde und Sachverstand in einem kombinierten Kurs}

Die meisten betroffenen Ärzte wollen nicht nur die für ihre Facharztausbildung geforderte Sachkunde für dosisintensive Röntgenunter- 
6 Otto M, Zeller W. Onlineprüfung Sachverstand Strahlenschutz. Schweiz Ärztezeitung 2005; 86(17):1014-16. suchungen erwerben, sondern auch den Sachverstand, entweder weil sie eine Praxiseröffnung bzw. -übernahme planen, oder weil sie sich die Möglichkeit für eine zukünftige Betriebsbewilligung schaffen wollen. Diesen Kandidaten kommt eine Übereinkunft zwischen BAG und den Fachgesellschaften entgegen. Zusätzlich zur vorgeschriebenen Anzahl von bestimmten Röntgenuntersuchungen (je nach Facharztgebiet) muss nur ein Kurs von fünf Tagen Dauer mit Prüfung besucht werden. Damit werden sowohl die Sachkunde wie auch der Sachverstand erworben.

Einige Zahlen von den bisherigen Kursen In den Jahren 1997 bis 2004 wurden Kurse für die Erlangung des Sachverstandes im Niedrigdurchgeführt: an den Universitätsspitälern in Lausanne/Genf, Bern, Basel und Zürich, am Kantonsspital Frauenfeld, an der PSI-Strahlenschutz-

Tabelle 1

Übersicht über die Strahlenschutzkurse in Basel und Lausanne.

\section{Radiol. Physik, Basel}

25

Anzahl Kurse 1997-2005

Anzahl Teilnehmer total 1997-2005

Anzahl Teilnehmer für die Sachkunde (ab 2003)
PRORAME, Lausanne

411

62 dosisbereich an folgenden Ausbildungsstätten

schule in Villigen und im Kanton Tessin. Seit 2003 werden zudem vom BAG anerkannte kombinierte Ausbildungskurse für die Sachkunde wie auch für den Sachverstand im dosisintensiven Bereich von der Abteilung Radiologische Physik des Universitätsspitals Basel und von der Stiftung PRORAME in Lausanne (Fondation pour la promotion de la radioprotection en médecine; Sekretariat: Institut universitaire de radiophysique appliquée, Lausanne) durchgeführt. Nach Angaben des BAG wurden von 1997 bis 2004 insgesamt 2629 Ärzte in Kursen zu Sachverständigen ausgebildet. Von PRORAME und von der Radiologischen Physik in Basel liegen die Zahlen in Tabelle 1 vor. Zusätzlich absolvierten 1772 Ärzte die alleinige Prüfung [6].

Für die Erlangung des Sachverstandes (inkl. Übergangsregelung bis September 2004) bzw. der Sachkunde für dosisintensive Röntgenuntersuchungen nahmen in den letzten beiden Jahren an den Kursen in Basel und Lausanne vor allem Fachärzte gemäss Tabelle 2 teil.

Als Lehrkräfte für die 20 Theorie- und 20 Praktikalektionen in den jeweiligen Kursen stehen Medizin-Physiker, Strahlenbiologen, Radiologen, MTRA und Strahlenschutzinspektoren des BAG zur Verfügung; diese Einsätze werden ausserhalb der Arbeitszeit geleistet. Zusätzliche Personen stellen die Organisation und die Infrastruktur sicher. Als Problem erweist sich zunehmend die Verfügbarkeit von geeigneten Röntgengeräten für die Praktika. Es sollten dabei nach Möglichkeit die Anforderungen an die unterschiedlichen Facharztgebiete berücksichtigt werden können.

Anteil der Teilnehmer der Strahlenschutzkurse in Basel und Lausanne in den einzelnen Fachgebieten.

\begin{tabular}{lll}
\hline Facharztgebiete & Radiol. Physik, Basel & PRORAME, Lausanne \\
\hline Allgemeinmedizin & $27 \%$ & $25 \%$ \\
\hline Innere Medizin & $12 \%$ & $15 \%$ \\
\hline Orthopädie & $11 \%$ & $16 \%$ \\
\hline Rheumatologie & $11 \%$ & $5 \%$ \\
\hline Physikalische Medizin/Rehabilitation & $9 \%$ & $2 \%$ \\
\hline ORL & $1 \%$ & $10 \%$ \\
\hline Gastroenterologie & $7 \%$ & $4 \%$ \\
\hline Urologie & $2 \%$ & $8 \%$ \\
\hline Chirurgie & $3 \%$ & $7 \%$ \\
\hline Pneumologie & $4 \%$ & $2 \%$ \\
\hline Anästhesie & $4 \%$ & $2 \%$ \\
\hline Pädiatrie & $3 \%$ & $2 \%$ \\
\hline Kardiologie & $2 \%$ & $2 \%$ \\
\hline Angiologie & $1 \%$ & - \\
\hline Gynäkologie & $1 \%$ & - \\
\hline Neurochirurgie & $1 \%$ & - \\
\hline übrige & $1 \%$ & - \\
\hline
\end{tabular}

\section{Erfahrung und Schlussfolgerungen aus den Kursen}

Wie die Erfahrung zeigt, ist es für die Qualität der Strahlenschutzausbildung von Ärzten entscheidend, dass diese an einer Ausbildungsstätte mit der notwendigen Infrastruktur für die praktische Ausbildung und mit erfahrenen Dozenten aus der Praxis durchgeführt wird. Dies gilt insbesondere für die Ausbildung von Fachärzten, welche dosisintensive oder interventionelle diagnostische Anwendungen durchführen. Als Voraussetzung für die Anerkennung einer Ausbildungsstätte stellt das BAG Anforderungen, die für bestimmte Anwendungen nur in einem Spital mit den entsprechenden Ausbildungsmöglichkeiten und Fachärzten zu erbringen sind.

Die oft gefürchtete Prüfung kann unterschiedlich durchgeführt werden. In Basel werden zu Beginn des Kurses die (etwa 20) Prüfungsfragen abgegeben. Die Teilnehmer haben dann während des Kurses Gelegenheit, die Antworten in den Lektionen oder aus den abgegebenen 
Lehrmitteln zu finden bzw. sie an den Praktikumsplätzen zu erarbeiten. Damit wird bezweckt, dass sich die Teilnehmer mit dem Stoff auseinandersetzen und untereinander sowie mit den Lehrkräften die Probleme diskutieren. Die Teilnehmer arbeiten jeweils mit grossem Engagement an den Antworten. Die Fragen sind sehr unterschiedlich und können mit Wissen, Berechnungen, Überlegungen und praktischen Erfahrungen gelöst werden. Von den 680 Teilnehmern in Basel hat ein einziges Mal ein Kandidat die Anforderungen nicht erfüllt. Die Prüfung kann einmal wiederholt werden.

Bei den Kursen der Stiftung PRORAME wird am Ende des Kurses eine Prüfung bestehend aus einem Multiple-Choice-Fragebogen und einer Patientenpositionierung organisiert. Auch hier sind die Motivation und die Mitwirkung der Kandidaten sehr gross. Von den Teilnehmern musste nur in einem Fall die Prüfung wiederholt werden.

Die Evaluation der Kurse durch die Teilnehmer zeigt ein relativ konstantes Bild. Zu Beginn erklärt jeweils eine Minderheit der Kursteilnehmer, sie wären motiviert. Die Hälfte bezeichnet sich immerhin als mässig motiviert. Am Ende der Kurse erklärt sich jeweils der weitaus grösste Teil als zufrieden bis begeistert. Die Erwartungen der Teilnehmer wurden nach ihren eigenen Angaben jeweils fast vollständig erfüllt. Das mit Abstand beliebteste Unterrichtsgebiet ist die praktische Einstelltechnik. Diese bildet mit 10 von 40 Lektionen denn auch ein eindeutiges Schwergewicht. Gute Noten (über $75 \%$ ) erhalten jeweils auch: praktische Übungen in Röntgendiagnostik, Bildqualität (Theorie und Praktika), Bildverarbeitung und -dokumentation (Theorie und Praktika), Strahlenphysik und Dosimetrie (Theorie) und Indikationsstellung (Theorie). Im Mittelfeld (66-75\%) liegen: Funktionsweise von Röntgenanlagen (Theorie und Praktika), Strahlenbiologie (Theorie) und Konstanzprüfung (Praktika). Am wenigsten beliebt (50-65\%) sind hingegen die Lektionen, wo es um die Pflichten als Strahlenschutzverantwortlicher geht: Einrichtung Röntgenbetrieb (Theorie), Konstanzprüfungen (Theorie), Strahlenschutz des Patienten und des Personals (Theorie und Praktika) sowie Rechtsgrundlagen Strahlenschutz (Theorie). Das liegt teilweise natürlich auch an den Lehrkräften. Die Lehrkräfte sind durchwegs motiviert, was die Kursteilnehmer in der Evaluation jeweils auch besonders erwähnen. Die Zusammenarbeit zwischen Ärzten und MTRA in den Praktika ist sehr gut und fast immer problemlos. Die für den Kurs ausgewählten MTRA sind sehr kompetent und können den Ärzten ihre grosse Erfahrung vermitteln. Das ist gut so, denn die MTRA sind bekanntlich die Fachpersonen für die praktische Röntgentätigkeit.

\section{Blick in die Zukunft}

Nachdem nun Erfahrungen über die Anforderungen und Bedürfnisse der Kursteilnehmer über einige Jahre hinweg vorhanden sind, ist man in der Lage, die Ausbildungsziele und -inhalte zu überprüfen und sie anzupassen. Diese wurden im Jahre 1996 von einer breitabgestützten Arbeitsgruppe unter der Leitung des BAG aufgestellt [4].

Ob dem oft vorgebrachten Wunsch nach Verkürzung der Kursdauer entsprochen werden kann, ist fraglich, wenn der steigende Umfang der Anwendungsbedürfnisse und der technischen Möglichkeiten weiterhin abgedeckt werden soll. Für die Festsetzung der Anforderungen an die Sachkunde für dosisintensive Röntgenuntersuchungen sind grundsätzlich die Fachgesellschaften zuständig, in Absprache mit dem BAG.

Es gibt auch bereits Anfragen von Kursabsolventen für Wiederholungskurse. Repetitionskurse könnten beispielsweise ein bis zwei Tage dauern und alle fünf Jahre angeboten werden. Dabei könnte über neue Technologien sowie aktuelle Strahlenschutzprobleme und neue Weisungen bzw. Empfehlungen orientiert werden.

Ein ungelöstes Problem scheint die praktische Ausbildung zur Erlangung der Sachkunde für dosisintensive Untersuchungen $\mathrm{zu}$ sein, insbesondere im Gebiet der Allgemeinmedizin und teilweise der Inneren Medizin. Verschiedene Kursteilnehmer bemängeln die ungenügende Anzahl von Ausbildungsplätzen, um die im Weiterbildungsprogramm der FMH geforderte Zahl von Röntgenuntersuchungen unter Anleitung in der Praxis durchführen zu können.

Infolge Auflösung der Radiologischen Physik am Universitätsspital Basel werden für verschiedene Fachrichtungen zurzeit nur noch durch die Stiftung PRORAME vom BAG anerkannte Kurse angeboten. Eine neue Ausbildungsstätte für deutschsprachige Ärzte muss gefunden und aufgebaut werden. 